\title{
ПРИМЕНЕНИЕ КОНТРОЛЬНЫХ КАРТ ШУХАРТА ПРИ ПОДГОТОВКЕ ОБЗОРА КАЧЕСТВА ЛЕКАРСТВЕННЫХ ПРЕПАРАТОВ
}

\author{
(С) Мцариашвили М.Р. ${ }^{1}$, Гармонов С.Ю. ${ }^{1}$, Садыкова Е.Р. ${ }^{2}$, Егорова С.Н. ${ }^{3}$ \\ ${ }^{1}$ Кафедра аналитической химии, сертификации и менеджмента качества Казанского национального \\ исследовательского технологического университета, Казань; ${ }^{2}$ отдел контроля качества ОАО \\ «Татхимфармпрепараты», Казань; ${ }^{3}$ кафедра фармации Казанского государственного медицинского \\ университета, Казань \\ E-mail: m.r.mtsariashvili@yandex.ru
}

В работе представлено применение контрольных карт Шухарта при написании ежегодного обзора качества лекарственных препаратов для анализа стабильности производственного процесса и применение показателей возможностей процесса с целью улучшения производственного процесса. Авторами представлена последовательность применения контрольных карт Шухарта для выявления различных случаев состояний процессов и расчет индексов воспроизводимости и индексов пригодности, позволяющая интерпретировать исходные данные для эффективной оценки качества лекарственного препарата и принятия управленческих решений. На примере некоторых твердых, жидких и мягких лекарственных форм при помощи оценки статистических характеристик, используемых при подготовке обзора их качества, определили возможность оптимизации процесса производства и обосновали необходимые управленческие решения, подтвердили стабильность процесса производства, выявили необходимость стабилизации среднего показателя, установили причины неуправляемости процесса производства.

Ключевые слова: качество лекарственных препаратов, обзор качества, GMP, статистические методы, управление процессом.

\section{APPLICATION OF SHEWHART CONTROL CHARTS WHEN REVIEWING THE QUALITY OF MEDICINAL DRUGS \\ Mtsariashvili M.R. ${ }^{l}$, Garmonov S.Yu. ${ }^{\prime}$, Sadykova E.R. ${ }^{2}$, Egorova S.N. ${ }^{3}$}

${ }^{1}$ Department of Analytical Chemistry, Certification and Quality Management of Kazan National Research Technological University, Kazan; ${ }^{2}$ Department of Quality Control of Public Corporation «Tatkhimpharmpreparaty», Kazan; ${ }^{3}$ Pharmacy Department of Kazan State Medical University, Kazan

The paper presents the application of Shewhart control charts in the annual review of medicinal products quality in order to analyze the production process stability and to use the indicators of process capability for the purpose of its enhancement. The authors presented the sequence of applying Shewhart control charts to identify the various states of manufacture processes and calculate reproducibility and suitability indices, enabling to interpret the input data for effective assessment of drug quality and proper management decisions. By example of some solid, liquid, and soft medicinal forms and by means of assessing the statistical characteristics used in drug quality review, we identified the possibilities of optimizing the production process, grounded the required management dicisions, confirmed the production process stability, identified the need for stabilizing the mean value, and established the causes of the production process uncontrollability.

Keywords: drug quality, quality review, GMP, statistical techniques, process control.

Для эффективной оценки качества лекарственных препаратов (ЛП) и принятия управленческих решений с целью постоянного улучшения эффективности процессов и качества ЛП необходим тщательный анализ параметров и показателей, заявленных в стратегии контроля.

Обзор качества (ОК) лекарственных средств (ЛС) - это регулярно проводимая и оформленная документально оценка качества всех произведенных фармацевтическим предприятием ЛС «с целью подтверждения постоянства имеющегося процесса, соответствия действующим спецификациям как на исходные сырье, так и на готовую продукцию, для выявления тенденции и установления возможности улучшения продукции и процесса» $[1,4]$.
Одним из широко используемых в управлении качеством продукции методов выявления факторов, влияющих на процесс производства ЛП, с целью их снижения и устранения, является применение контрольных карт Шухарта [2].

Целью исследования являлось применение статистических методов для анализа технологических процессов производства лекарственных препаратов на примере некоторых твердых, жидких и мягких лекарственных форм, а также оценка статистических характеристик, используемых при подготовке обзора их качества. 


\section{МАТЕРИАЛЫ И МЕТОДЫ ИССЛЕДОВАНИЯ}

Проведен обзор качества 18 товарных серий ЛП «Ацикловир таблетки 200 мг», 22 товарных серий ЛП «Амброксол сироп 3 мг/мл», 40 товарных серий ЛП «Метилурацил, мазь для наружного применения 25\%».

Первоначальная информация интерпретировалась при помощи контрольных карт Шухарта, в которых представлены результаты значений показателя качества в соответствии с номером товарной серии. Использование контрольных карт индивидуальных значений показателей качества и скользящих размахов (X- и MR-карты) обусловлено ограниченностью выборки по итогам контроля качества готовой продукции и параметров процесса производства ЛП. Оценка возможностей процесса осуществлялась расчетом комбинаций индексов:

- воспроизводимости процесса, оценивающего возможности удовлетворять технический допуск без учета положения среднего значения и применяемый для стабильных по разбросу процессов $C_{p}$;

- воспроизводимости оценивающего возможности процесса, удовлетворять технический допуск с учетом фактического положения среднего значения и применяемый для стабильных и по разбросу, и по настройке процессов $C_{p k}$;

- пригодности процесса удовлетворять технический допуск без учета положения среднего значения, применяемый для процессов, стабильность которых по разбросу не подтверждена $P_{p}$;

- пригодности процесса удовлетворять технический допуск с учетом положения среднего значения, применяемый для процессов, стабильность которых по разбросу не подтверждена $P_{p k}$ [3].

\section{РЕЗУЛЬТАТЫ ИССЛЕДОВАНИЯ И ИХ ОБСУЖДЕНИЕ}

В фармакопейных статьях предприятия на твердые лекарственные формы (таблетки) одним из важных показателей качества является средняя масса таблетки. На примере производства ЛП «Ацикловир таблетки 200 мг» была построена контрольная карта скользящих размахов для 18 товарных серий (рис. 1).
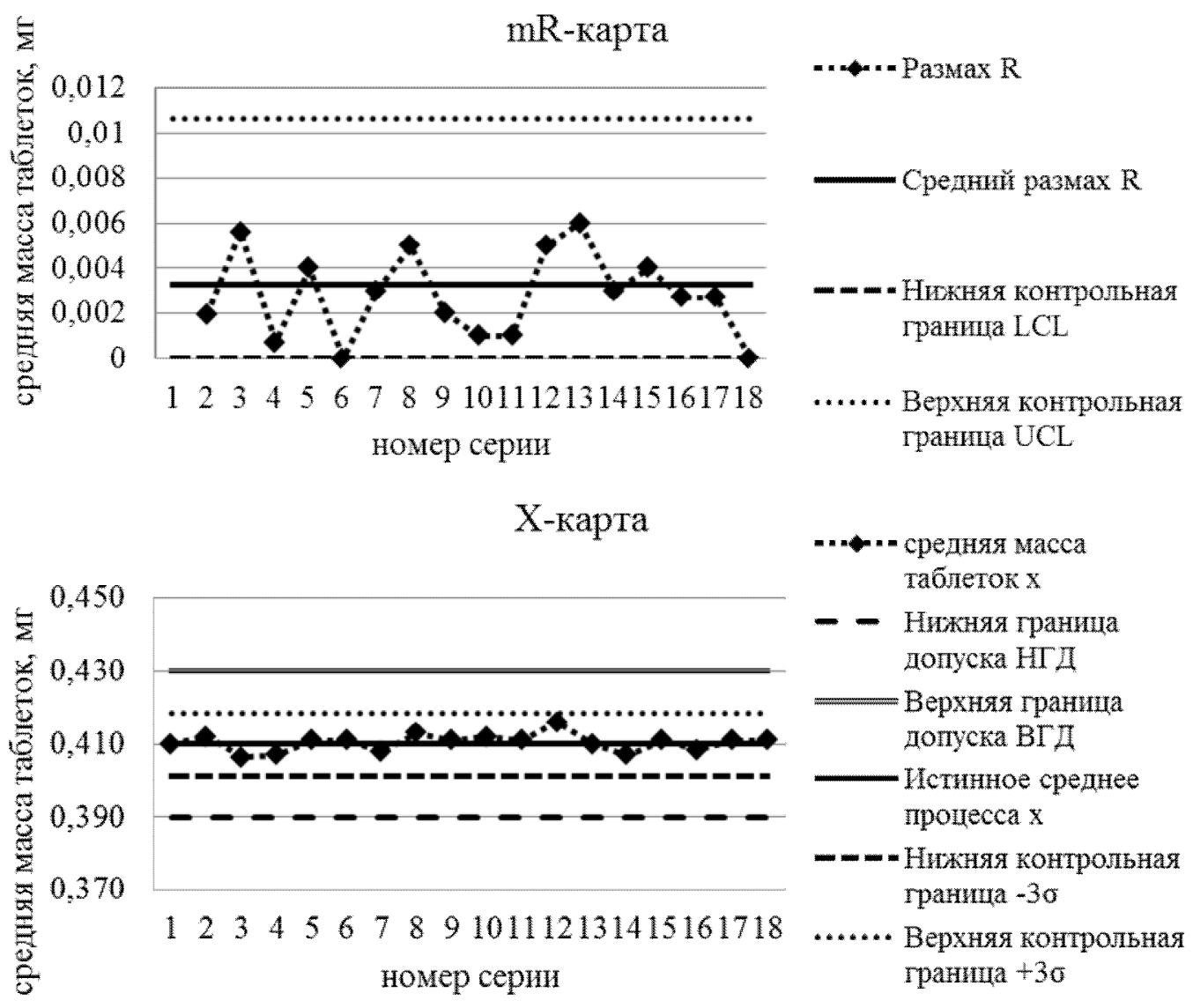

Рис. 1. MR- и X-карты распределения значений средней массы в готовом продукте. 


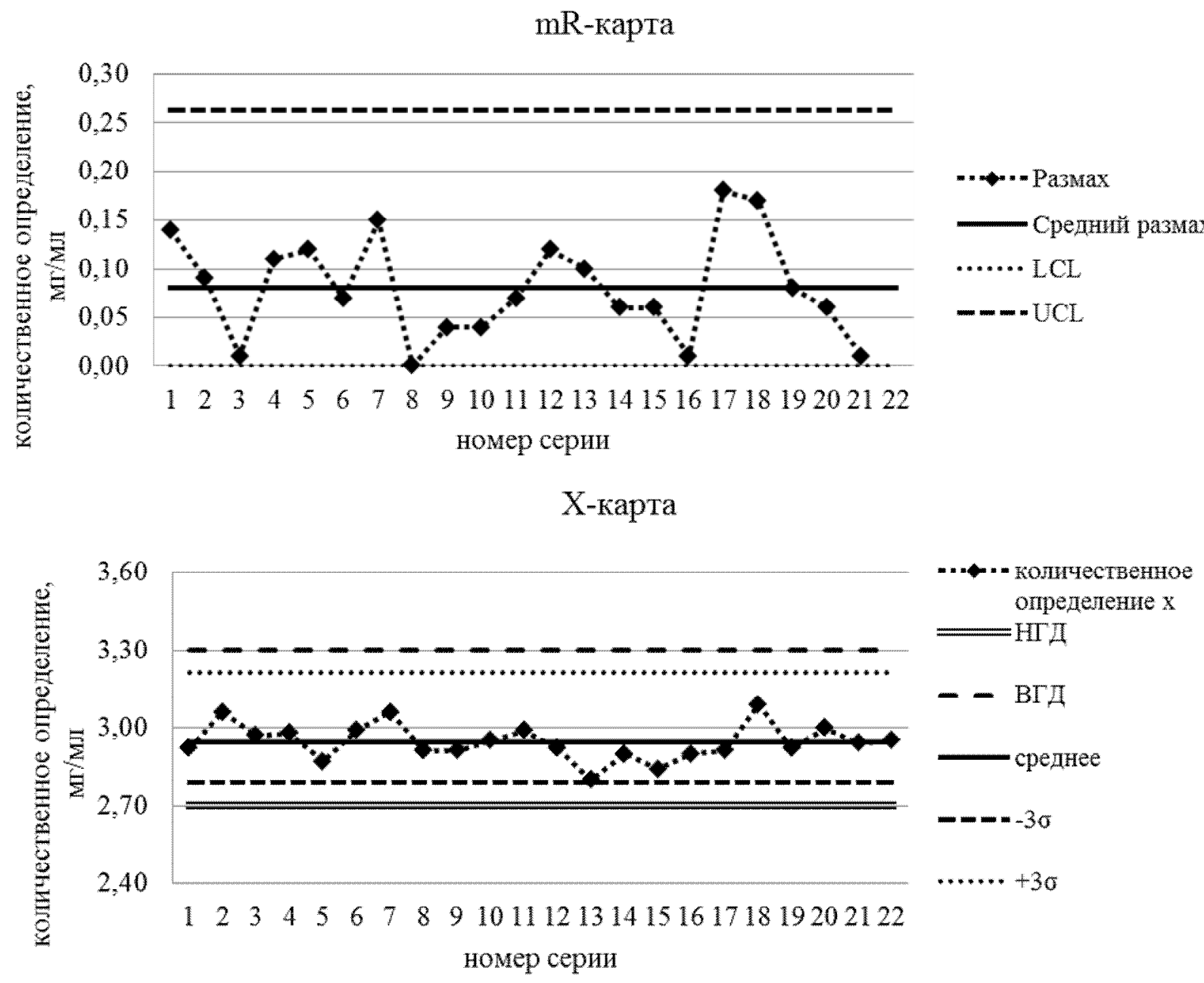

Pис. 2. MR- и X-карты распределения значений количественного содержания амброксола гидрохлорида.

Анализ контрольных MR- и X-карт (рис. 1) показал, что процесс производства ЛП «Ацикловир таблетки 200 мг» по показателю «средняя масса таблеток» настроен на центр поля допуска с учетом границ спецификации. Распределение значений сосредоточено относительно истинного среднего, равного 0,410 г, характеризуется высокой воспроизводимостью от серии к серии и можно сделать вывод, что процесс стабилен.

Для процесса, находящегося в статистически управляемом состоянии, необходимо оценить влияние только общих причин изменчивости [3]. Для данного распределения значений средней массы таблеток выборочное стандартное отклонение составило 0,003 , что подтверждает отсутствие влияния общих причин изменчивости. Поскольку в результате оценки стабильности процесса подтверждена его статистическая управляемость, то расчет показателей возможностей процесса производили при помощи индексов $\mathrm{C}_{\mathrm{p}}$ и $\mathrm{C}_{\mathrm{pk}}$. [3]. Рассчитанные значения индексов составили 2,31, что свидетельствует о воспроизводимости процесса $\left(\mathrm{C}_{\mathrm{p}}>1,33\right)$, в основе которого лежит стабильное нормальное распределение значений данного показателя качества. Равенство значений индексов характеризует оптимальную настройку процесса на номинальное значение показателя качества.

На примере анализа процесса производства ЛП «Аброксол сироп 3 мг/мл» провели статистическую обработку показателя качества готовой продукции «количественное содержание».

Анализ контрольных MR- и X-карт (рис. 2) готовой продукции показал, что распределение значений количественного содержания амброксола гидрохлорида стабильно по разбросу, но не стабильно по положению среднего значения, которое составляет - 2,9 мг/мл. Необходимо отметить, что имеется серия, находящаяся на контрольной границе. Для данного процесса необходимо оценить как собственную изменчивость процесса, так и полную изменчивость. Для полученного распределения значений показателя качества выборочное стандартное отклонение составило 0,07 как для полной, так и для внутренней изменчивости процесса, что говорит о более значимом влиянии общих причин изменчивости и требует фундаментальных изменений процесса. 

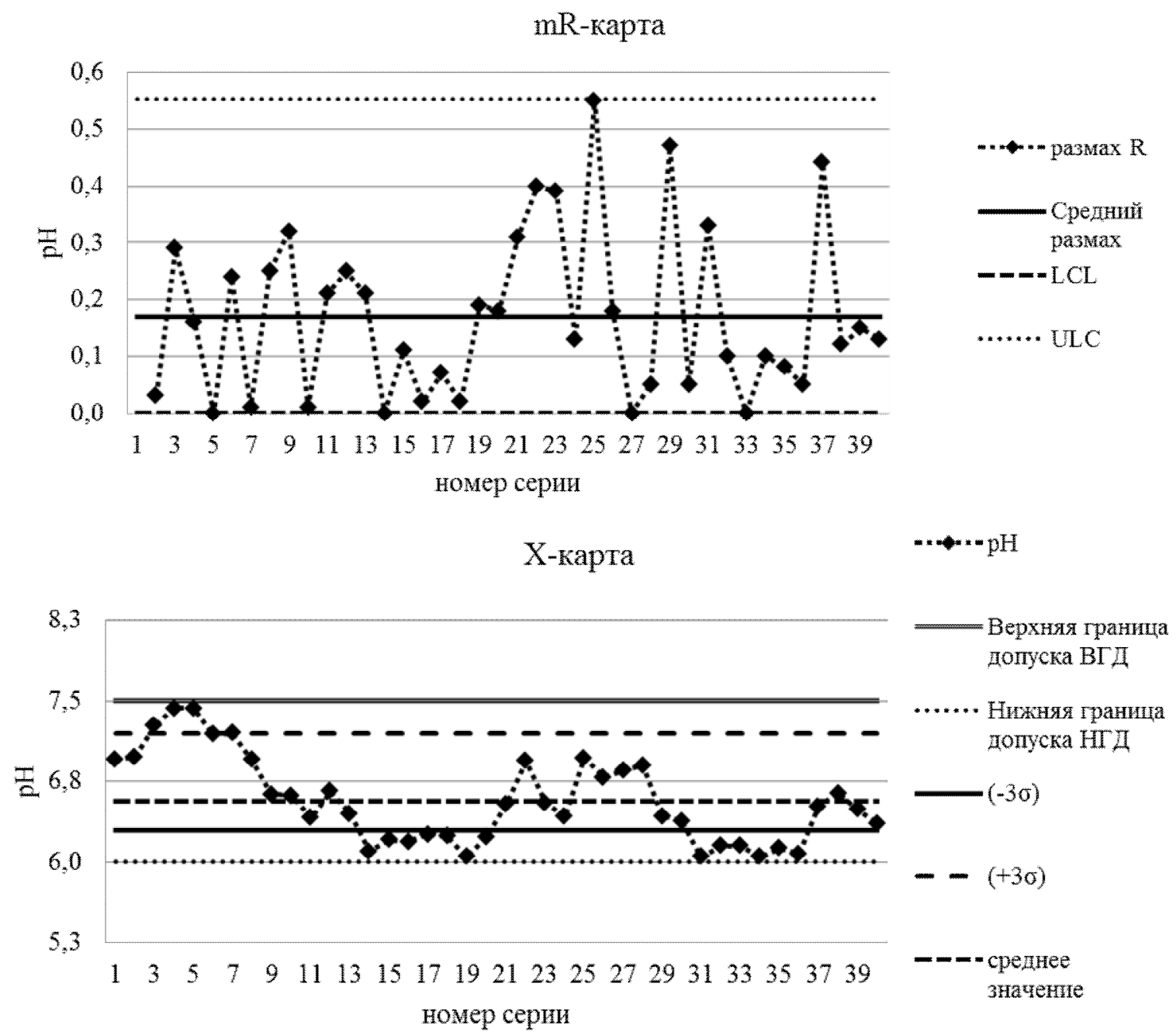

Рис. 3. MR- и X-карты распределения значений $\mathrm{pH}$.

Расчет показателей возможностей процесса производили при помощи индексов $\mathrm{C}_{\mathrm{p}}, \mathrm{P}_{\mathrm{p}}$ и $\mathrm{P}_{\mathrm{pk}}$. Рассчитанное значение $\mathrm{C}_{\mathrm{p}}$ составило 1,4 , при этом процесс стабилен по разбросу, однако не стабилен по настройке и среднее смещено к нижней границе допуска. Процесс приготовления амброксола сиропа нельзя оставлять без изменений, о чем свидетельствует индекс $\mathrm{P}_{\mathrm{pk}}$, равный 1,16 . Необходимо предпринять меры, направленные на обеспечение стабильности среднего. Была выявлена необходимость внесения изменений в технологию производства, а именно, необходимость пересмотрения регламентированных значений температуры растворения ингредиентов и регламентирования параметров перемешивания для стабилизации показателей качества готовой продукции.

На примере анализа процесса производства ЛП «Метилурацил, мазь для наружного применения $10 \%$ провели обработку показателя качества готовой продукции - значения «рН».

Анализ контрольных MR- и X-карт (рис. 3) показал, что процесс производства препарата по показателю «рН» не стабилен по разбросу и положению среднего. Процесс находится в статисти- чески неуправляемом состоянии, 18 серий вышли за контрольные границы. Среднее значение показателя смещено к нижней границе допуска. Определили полную изменчивость процесса, рассчитав выборочное стандартное отклонение, которое составило 0,4. Оценку показателей возможностей процесса производили при помощи индексов $\mathrm{P}_{\mathrm{p}}$ и

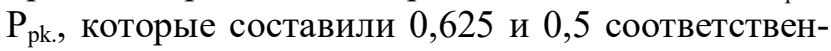
но. Возможности процесса неприемлемы, значения индексов слишком малы. Необходимо принять меры, направленные на обеспечение стабильности. Было установлено влияние материалов, а именно, данная изменчивость обусловлена использованием сырья разного качества.

Проведен сравнительный анализ производителей вазелина, входящего в состав мазевой основы, с целью выявления причин вариабельности. Качество сырья соответствовало установленным требованиям (табл. 1).

Каждая загрузка вазелина, необходимая для производства товарной серии метилурацила, мази для наружного применения, проходит процесс стерилизации. Ниже представлены графики изменения значений $\mathrm{pH}$ вазелина после прохождения процесса стерилизации. 
Показатели качества вазелина

\begin{tabular}{|c|c|c|c|c|}
\hline $\begin{array}{c}\text { Номер } \\
\text { серии }\end{array}$ & $\begin{array}{c}\mathrm{pH}, \\
6,5-7,5\end{array}$ & $\begin{array}{c}\text { Температура плавления } \\
\text { (каплепадения) } \\
(37-60),{ }^{\circ} \mathrm{C}\end{array}$ & $\begin{array}{c}\text { Кислотное число, } \\
\text { не более } 0,1 \text { мг }\end{array}$ & $\begin{array}{c}\text { Кинематическая вязкость при } 60^{\circ} \mathrm{C}, \\
\text { не менее } 16 \text { сСт }\end{array}$ \\
\hline \multicolumn{5}{|c|}{ Вазелин "SOSOL WAX GMBH", Германия } \\
\hline 1 & 6,6 & 56 & - & - \\
\hline \multicolumn{5}{|c|}{ 3AO "Mедхим", Россия } \\
\hline 2 & 6,7 & 58 & 0,03 & 31,4 \\
\hline 3 & 6,8 & 58 & 0,06 & 54 \\
\hline 4 & 7,2 & 60 & 0,03 & \\
\hline
\end{tabular}

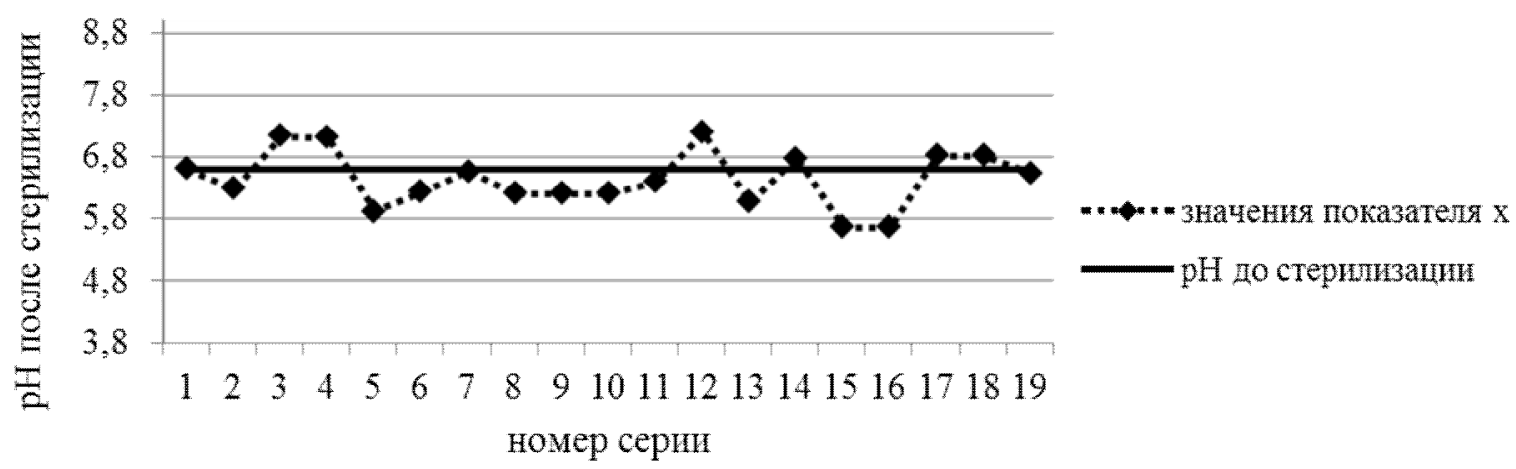

Рис. 4. График изменения показателя $\mathrm{pH}$ вазелина после стерилизации (производитель "SOSOL WAX GMBH").

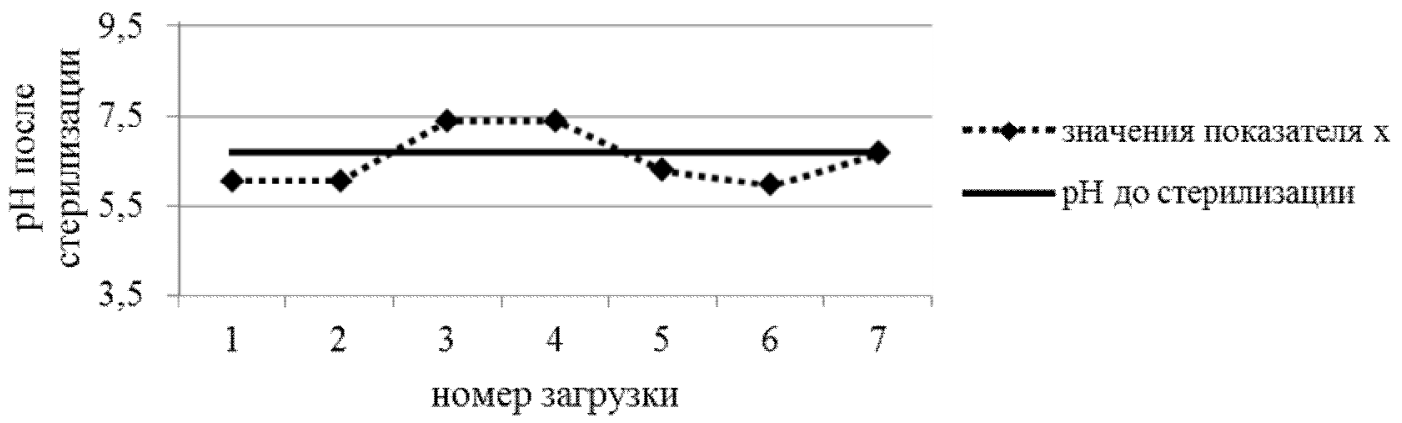

Рис. 5. График изменения показателя $\mathrm{pH}$ вазелина (серия 2) после стерилизации (производитель ЗАО «Медхим»).

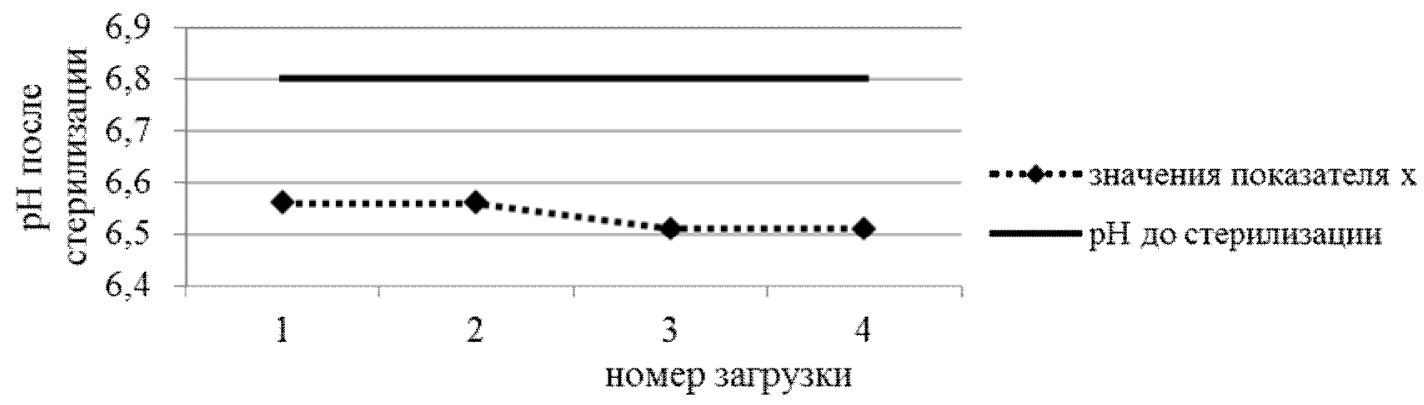

Рис. 6. График изменения показателя $\mathrm{pH}$ вазелина (серия 3) после стерилизации (производитель $3 \mathrm{AO}$ «Медхим»). 


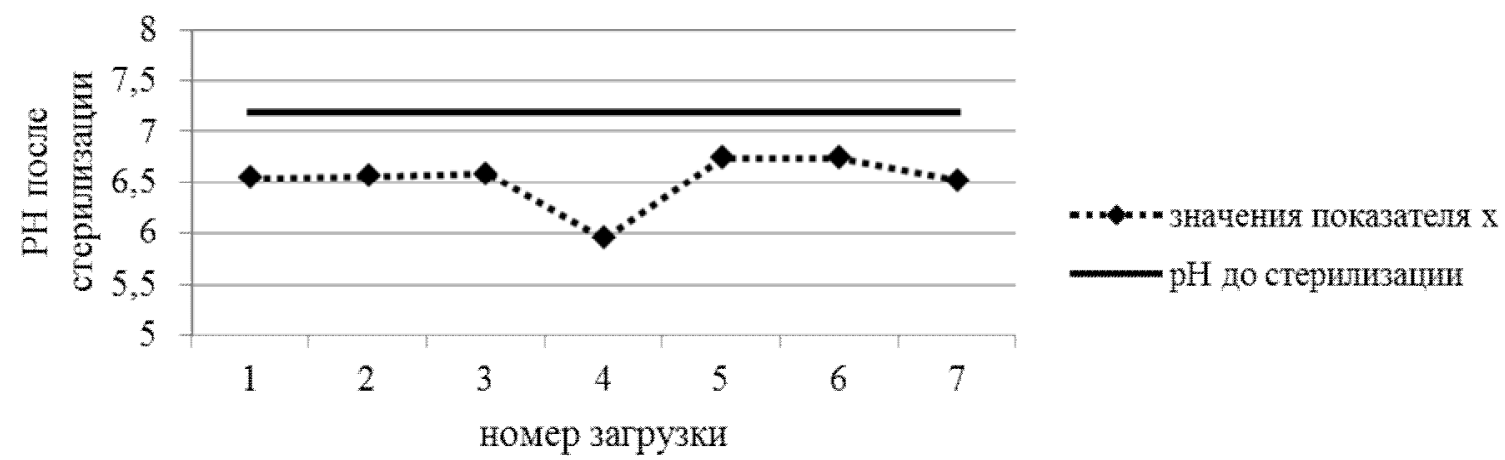

Рис. 7. График изменения показателя $\mathrm{pH}$ вазелина (серия 4) после стерилизации (производитель ЗАО «Медхим»).

Как видно из данных, представленных на рис. 4, после проведения стерилизации значения показателя $\mathrm{pH}$ вазелина меняются, распределение данных значений сосредоточено относительно значения рН до стерилизации. Отсюда следует, что субстанция данного производителя стабильна, так как резкие скачки рН отсутствуют.

Анализ графиков (рис. 5-7) изменения значений $\mathrm{pH}$ производителя «Медхим» показал, что после процесса стерилизации вазелина всех трех серий наблюдается понижение значений $\mathrm{pH}$.

Анализ данных показателя качества «рН вазелина после стерилизации» двух производителей "SOSOL WAX GMBH", Германия (1 серия) и ЗАО «Медхим», Россия (3 серии) показал, что значения $\mathrm{pH}$ вазелина производителя ЗАО «Медхим» после стерилизации снижаются, что повлияло на качество готовой продукции. Наиболее подходящим производителем вазелина для состава мазевой основы для ЛП «Метилурацил, мазь для наружного применения 10\%» является "SOSOL WAX GMBH", Германия.

Таким образом, с использованием контрольных карт Шухарта при подготовке обзора качества для различных состояний процесса определили возможность оптимизации процесса производства и обосновали необходимые управленческие решения. При этом подтвердили стабильность процесса производства
ЛП «Ацикловир таблетки 200 мг» по показателю «средняя масса таблеток», выявили необходимость стабилизации среднего показателя «количественное определение» для ЛП «Амброксол сироп 3 мг/мл» и установили причины неуправляемости процесса производства ЛП «Метилурацил, мазь для наружного применения $25 \%$ » по показателю $\mathrm{pH}$.

\section{ЛИТЕРАТУРА}

1. ГОСТ Р 52249-2009. Правила производства и контроля качества лекарственных средств. - Введ. 2009-06-08. - М. : Изд-во стандартов, 2009.

2. ГОСТ Р ИСО 7870-2-2015. Статистические методы. Контрольные карты. Часть 2. Контрольные карты Шухарта. - Введ. 2015-10-06 М. : Стандартинформ, 2015.

3. ГОСТ Р 50779.44-2001. Статистические методы. Показатели возможностей процессов. Основные методы расчетов. - Введ. 2002-07-01 - М. : Изд-во стандартов, 2001.

4. Приказ Министерства промышленности и торговли от 14 июня 2013 г. № 916 «Об утверждении Правил организации производства и контроля качества лекарственных средств» [Электронный ресурс] // Гарант.ру. - Режим доступа:

http://www.garant.ru/products/ipo/prime/doc/7035119 8/\#ixzz3tBOfaRPZ, свободный (25.05.2016). 